\title{
La tecnología de recuperación y protección contra el desgaste está en el rociado térmico
}

\section{The technology against the wearing is the thermal spray}

\author{
José Luddey Marulanda Arévalo ${ }^{1}$, José Luis Tristancho Reyes ${ }^{2}$, Héctor Álvaro Gonzáles B. ${ }^{3}$ \\ 1,2Ph. D. Profesor Universidad Tecnológica de Pereira. La Julita-Pereira-Colombia. \\ ${ }^{3}$ MSc. Profesor Universidad Tecnológica de Pereira. La Julita - Pereira-Colombia. \\ Email: jlmarulanda@utp.edu.co,josetris@utp.edu.co
}

Recibido 7/02/14, aceptado 28/02/2014

\section{RESUMEN}

Los recubrimientos por rociado térmico están ayudando a controlar y disminuir los problemas superficiales que implican desgaste y corrosión. Los recubrimientos por rociado térmico sirven para mejorar características mecánicas, físicas y químicas, dando calidades superiores de funcionamiento a la superficie. La variedad de productos y capas que se pueden obtener por rociado térmico, son virtualmente ilimitadas. Las técnicas de rociado térmico son sistemas de combustión por llama, oxi-combustible de alta velocidad, detonación; sistemas de arco eléctrico, plasma con arco transferido y no transferido. Estos procesos son usados con éxito en el mundo desarrollado y vienen incrementando las inversiones industriales en este campo, y también es usado por instituciones de investigación para el desarrollo de nuevas aplicaciones y la evaluación de las propiedades físicas, químicas y mecánicas de estas capas protectoras. La protección y modificación de la superficie y sus propiedades, son una herramienta potente en la ingeniería moderna, en donde las nuevas aplicaciones de la tecnología de rociado térmico son una alternativa técnica, económica y competitiva para la industria. También, permite mejorar la productividad de los procesos, contribuyendo a aumentar la funcionalidad de partes que trabajan bajo condiciones severas de desgaste.

Palabras clave: Rociado térmico, Recubrimientos, Desgaste, Corrosión, Ingeniería de superficies.

\begin{abstract}
Thermal spray coatings are helping to control and reduce superficial problems involving wear and corrosion. Thermal spray coatings are profitable and serve to improve mechanical, physical and chemical performance giving superior qualities to the surface. The variety of products and layers that can be obtained by thermal spraying is virtually limitless. The techniques of thermal spraying are combustion systems by flame spray, high velocity Oxi-fuel, detonation and electric arc systems, plasma spray with transferred and not transferred arc. These processes are used successfully in the developed world and are increasing industrial investment in this field, and are also used by research institutes to develop new applications and to evaluate the physical, chemical and mechanical properties of protective coatings. The protection and surface modification and their properties, are a powerful tool of modern engineering, where, new applications of thermal spray technology is an alternative technical, economic and competitive in industry, Also, it can improve processes productivity, helping to increase the functionality of parts working under severe conditions of wear.
\end{abstract}

Keywords: Corrosion, Thermal Spray, Coatings, Wear, Corrosion, Surfaces Engineering.

\section{INTRODUCCIÓN}

Industrias como la aeroespacial, automotriz, química, de alimentos, de petróleos, minera entre otras, utilizan los recubrimientos aplicados por la técnica de rociado térmico para prolongar la vida útil de sus productos, aumentar su eficiencia de producción y reducir los costos de mantenimiento. Estos recubrimientos pueden ser un medio rentable para proteger la superficie de una pieza mecánica que esté sufriendo problemas de desgaste, corrosión a alta temperatura y acuosa, regulación térmica y degradación. Otras aplicaciones de los recubrimientos aplicados por 
rociado térmico comprenden la restauración de medidas, ajustándolas a tolerancias precisas, así como modificación de las propiedades térmicas y la fricción durante el contacto deslizante, ya que se puede aplicar una capa con propiedades diferentes al material base [1,2]. La ingeniería de superficies es una de las más importantes áreas de desarrollo, en la búsqueda de soluciones para el incremento en la vida útil de componentes de máquinas e instalaciones industriales, lo que ahorraría gran parte del consumo mundial de materiales, ya que se disminuiría el cambio de piezas. Esto es válido tanto para los trabajos que involucran nuevos proyectos, como para los trabajos de mantenimiento, que pretenden maximizar los resultados de confiabilidad y disponibilidad de equipos e instalaciones. En el mundo contemporáneo donde se busca mayor competitividad, hay dos factores de extrema relevancia: alta productividad y bajo impacto ambiental, que supone un compromiso con el medio ambiente y la conservación de los recursos no renovables [3, 4]. El rociado térmico se está utilizando en la fabricación de sensores avanzados de tensión, flujo de calor entre otros y para los sectores biomédicos, de energía, ambiental y aeroespacial. Éstos y un arsenal extenso de usos que emergen, se están aprovechando de las ventajas, capacidades y rentabilidad de la tecnología del rociado térmico para la reparación y fabricación de piezas en calderas, intercambiadores de calor, turbinas, extrusoras, pistones, cilindros de laminación, maquinaria agrícola entre otras. La confiabilidad y la eficiencia de los recubrimientos hace necesario que estén bien seleccionadas y aplicadas correctamente, para un funcionamiento exitoso. El uso de la tecnología de rociado térmico viene creciendo debido principalmente, a los altos costos de los materiales avanzados y a los crecientes requerimientos de ciclo de vida de los sistemas de alto desempeño, ya que se puede usar un material económico como substrato y aplicar un recubrimiento para protegerlo de la condición agresiva. La combinación resultante puede tener mejores propiedades físicas, químicas o costos más económicos que el uso de un solo metal o aleación. Esto ha llevado al rápido desarrollo de la tecnología de recubrimientos para el uso en diversas aplicaciones de ingeniería $[4,5]$.

En Colombia hay varias empresas dedicadas a la aplicación de recubrimientos metálicos y no metálicos por rociado térmico sobre distintos tipos de superficies, algunas son; Meticsa, Ferresolda, Metalitec, SYG Metalización en Bogotá, Tametco, Reneval en Cali y Metalizar en Medellín, siendo SAGER con sus productos Eutectic-Castolin el mayor distribuidor de aleaciones para el rociado térmico.

\section{ROCIADO TÉRMICO}

Los recubrimientos aplicados por la tecnología de rociado térmico son formados por la deposición de capas sucesivas de splats que provienen de la fusión del material de partida en forma de polvo o alambre (millones de partículas por $\mathrm{cm}^{2} / \mathrm{sec}$ ) que se aplanan y solidifican, resultando en una macro estructura conocida como lamela o splats la cual está anclada mecánicamente a las irregularidades de la superficie. La estructura típica de un recubrimiento por rociado térmico es la unión cohesiva de láminas del material rociado, en combinación con inclusiones de óxidos, micro grietas, partículas sólidas y porosidad [6, 7, 8]. Las características físicas y el comportamiento de las capas aplicadas dependen de las fuerzas cohesivas entre las láminas, el tamaño y la morfología de la porosidad, la aparición de grietas y defectos en la microestructura ultra fina y granulosa dentro de las mismas láminas. Una lámina resulta cuando una gota del material fundido, de diez a cien micras en diámetro, se funde en la llama e impacta sobre la superficie, donde se aplana y solidifica, con una velocidad de enfriamiento alrededor de $10^{6}-10^{8} \mathrm{~m} / \mathrm{s}$ [9]. La sumatoria de estas láminas forma el recubrimiento. Las características del recubrimiento son afectadas por la temperatura y la viscosidad de las láminas, tensión superficial, así como otras variables tales como la velocidad de enfriamiento, criterios de solidificación, nucleación y crecimiento de cristales, formación de la fase, entre otras. Los aspectos de formación y solidificación de las láminas, son complejos y están correlacionados, además la forma de las láminas, es influenciada por el ángulo de aspersión y tiene un fuerte efecto en las características del recubrimiento, tales como porosidad, eficiencia y micro dureza [10, 11].

Para la aplicación del recubrimiento por rociado térmico se usa una pistola que funde el material, en forma de polvo, varilla o alambre, utilizando un gas para propulsarlo hacia el substrato, creando una superficie totalmente nueva. Para obtener buena adherencia del material proyectado sobre el metal base, se debe realizar una preparación previa eliminando todo tipo de contaminantes, manteniendo la rugosidad superficial, debido a que el principal mecanismo de adherencia del recubrimiento al substrato es el de anclaje mecánico, además esta preparación depende del espesor del recubrimiento que se desea obtener, de la naturaleza del material proyectado y de la forma de la pieza a recubrir. La adecuada preparación del substrato antes de la aplicación del revestimiento es la etapa más crítica que influencia la resistencia de unión y adhesión del recubrimiento al substrato [4, 12]. El principio de funcionamiento es observado en la figura 1 .

Para la obtención de recubrimientos de calidad, con el desempeño deseado en servicio, es necesario un control riguroso de las diversas etapas del proceso. En ocasiones, la falta de mantenimiento de los equipos es responsable por la mala calidad. Además la variación de los parámetros de aplicación del recubrimiento por rociado térmico, afecta las propiedades del recubrimiento como el tamaño y distribución de la porosidad, contenido de óxidos, ten- 
siones residuales, agrietamientos a escalas macroscópicas y microscópicas, las cuales tienen incidencia directa en la vida útil y la calidad de los recubrimientos [14, 15].

Figura 1. Esquema del proceso de rociado térmico por llama con polvos [4, 13].

Figure 1. Schematic process for flame thermal spraying powder $[4,13]$.

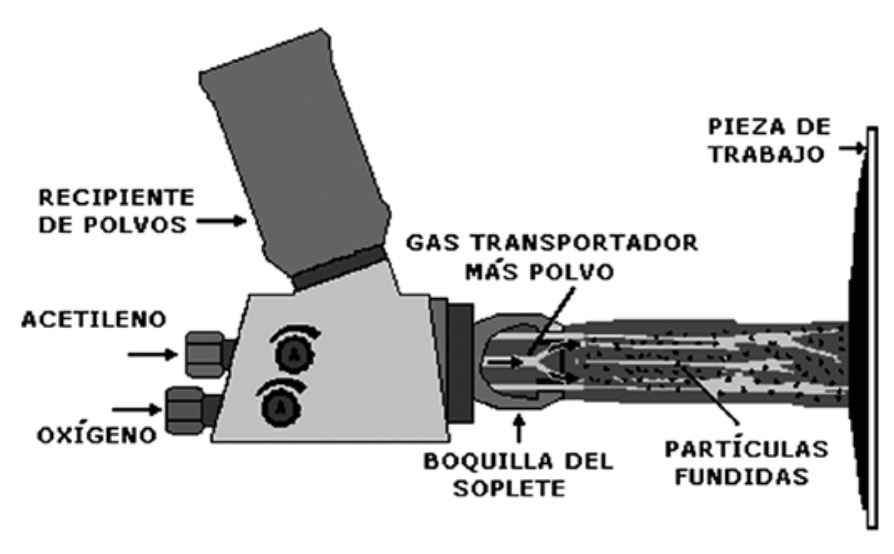

\section{PROCESOS DE ROCIADO TÉRMICO}

El proceso de rociado térmico es un tratamiento superficial, que no afecta la microestructura del metal base sobre el cual se deposita, ni tampoco hay una deformación excesiva; por esto las mejoras en las propiedades mecánicas son exclusivamente superficiales [16]. La apropiada selección del proceso de rociado térmico está determinado por el material deseado en el recubrimiento, requerimientos de desempeño, tamaño y forma de la pieza, así como factores económicos. Las técnicas de rociado térmico utilizan sistemas de combustión por llama, Oxi-Combustible de Alta Velocidad (HVOF- High Velocity Oxygen Fuel), detonación. Sistemas de arco eléctrico y arco eléctrico con propulsión y sistemas de plasma con arco transferido (PSP- Plasma Arc Spraying) y no transferido (PTA- Plasma Transferred Arc). El primer sistema utiliza gases combustibles como fuente de calor. El segundo y el tercer sistema lo constituyen los procesos que utilizan energía eléctrica como fuente de calor $[12,17]$.

La naturaleza del proceso de rociado térmico es de sinergia, ya que, existen diversas variables y componentes involucrados, los cuales, cuando actúan simultáneamente, producen un efecto mayor que cuando son considerados individualmente. En los procesos de rociado térmico ocurren dos etapas: (1) Atomización y (2) deposición. En la atomización tiene lugar el rompimiento del material líquido fundido en gotas y en la deposición se presentan dos momentos: (2.1) donde la gota está viajando e interactúa con el gas de atomización y (2.2) donde las gotas impactan e interactúan con el substrato. La unión entre el recubri- miento por rociado térmico y el substrato puede ser mecánica, metalúrgica, química, física o una combinación de estas formas. La adhesión es influenciada por una serie de factores, tales como el material del recubrimiento, condición del substrato, rugosidad de la superficie, limpieza, temperatura de la superficie antes, durante y después del rociado térmico y velocidad de impacto de la partícula. Las variaciones básicas del proceso de rociado térmico ocurren en los materiales utilizados, el método de calentamiento y el método de propulsión de los materiales hacia el substrato. Diversos metales, cerámicos, compuestos inter-metálicos, algunos polímeros y ciertos vidrios pueden ser depositados por uno o más de los procesos de rociado térmico, en diferentes tipos de substratos como metales, óxidos, cerámicos, vidrios y polímeros. Todos los materiales usados por rociado térmico no pueden ser aplicados sobre todos los substratos, ya que algunos requieren técnicas especiales $[4,18]$.

Sistemas de rociado térmico por combustión: Estos procesos utilizan la energía química de una llama de oxi-combustible como fuente de calor, para fundir los materiales del revestimiento en forma de polvo, varilla o alambre, estos materiales pueden ser metálicos y no metálicos con puntos de fusión menores a $2000{ }^{\circ} \mathrm{C}$. Además este grupo puede ser subdividido en: combustión por llama, oxicombustible de alta velocidad y detonación [19].

Rociado térmico por combustión convencional: Este proceso proyecta material fundido debido a la combustión del oxi-combustible, produciendo recubrimientos porosos y con limitaciones de adherencia, a cambio de ello, es la técnica más económica del mercado. Las antorchas de rociado térmico por llama, en su mayoría, pueden ser adaptadas para usar diversas combinaciones de gases, con el fin de obtener una optimización de los costos y la calidad del recubrimiento. Los gases comúnmente usados son acetileno, propano, gas metil-acetileno-propadieno e hidrógeno, combinados conjuntamente con oxígeno [20]. El acetileno es el gas más utilizado en función de obtener temperaturas de llama más altas, además de tener bajo costo. Prácticamente, es necesario solamente el cambio de la boquilla y de la "tapa de aire" (air cap) para adaptar la antorcha a las diferentes aleaciones, gases o tamaños de alambre. La relación de la mezcla oxígeno - combustible puede variar en la franja de 1:1 a 1.1:1, resultando en una atmósfera carburante $\mathrm{u}$ oxidante, respectivamente. La temperatura de la llama varía de $2.700^{\circ} \mathrm{C}$ a $3.100^{\circ} \mathrm{C}$ y la velocidad entre 80 $\mathrm{m} / \mathrm{s}$ a $100 \mathrm{~m} / \mathrm{s}[4,21,22]$.

Oxi-combustible de Alta Velocidad: Este proceso utiliza alta energía cinética y controla la salida de temperatura para producir recubrimientos densos de baja porosidad y adherencia que superan los 13.000 psi. Estos recubrimientos presentan bajos esfuerzos residuales permitiendo es- 
pesores hasta $12 \mathrm{~mm}$. Este proceso se desarrolló como una alternativa al proceso de rociado térmico por detonación, pero ahora compite con la técnica de rociado por plasma en algunas aplicaciones. Además de la calidad del recubrimiento y bajas tensiones residuales, otras ventajas incluyen alta eficiencia de depósito, menor sensibilidad al ángulo de rociado térmico y menos variables críticas del proceso [23, 24]. La figura 2 muestra esquemáticamente una antorcha de HVOF- High Velocity Oxygen Fuel.

Figura 2. Esquema de una antorcha de rociado térmico por oxi-combustible de alta velocidad (HVOF- High Velocity Oxygen Fuel) [4].

Figure 2. Schematic of a thermal spray torch for high velocity oxygen fuel. [4].

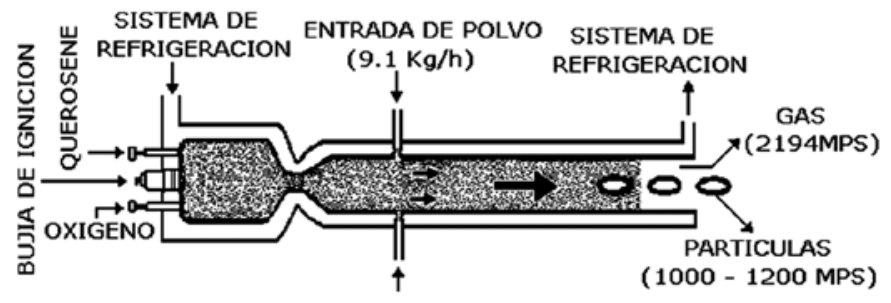

En este proceso se utiliza una mezcla de gas combustible con oxígeno de alta presión, que es quemado, generando un chorro de alta velocidad. El combustible puede ser propano, propileno, metil-acetileno-propadieno o hidrógeno. El material en forma de polvo es introducido en el cabezal utilizando nitrógeno como gas conductor. El combustible mezclado con el oxígeno es inyectado en la boquilla y encendido. Los productos de combustión son liberados y se expanden a través de la boquilla, donde las velocidades del gas pueden hacerse supersónicas. El polvo es introducido en la boquilla axialmente, siendo calentado y acelerado hacia fuera de la boquilla. La cámara de combustión y la boquilla son refrigeradas con agua $[25,26,27]$

Rociado térmico por detonación (D-Gun): El rociado térmico por detonación es un proceso de aplicación intermitente que utiliza la energía de la explosión de mezclas oxígeno-acetileno para calentar e impulsar los materiales en polvo hacia la superficie del substrato a ser recubierto. El depósito resultante es extremadamente duro, denso y con buena adherencia al substrato. El cañón de detonación consiste de un tubo dentro del cual se encuentra la cámara de combustión y es allí donde se introduce una mezcla de oxígeno, gas combustible (acetileno) y material en polvo del recubrimiento. Después de la ignición de la mezcla de gases, a través de una chispa eléctrica apropiada, una onda de detonación controlada, de alta presión y alta temperatura, acelera y calienta las partículas mientras las expulsa del tubo de la antorcha [28]. Un esquema de la antorcha de detonación es presentado en la figura 3.
Figura 3. Esquema de una antorcha de rociado térmico por detonación [13].

Figure 3. Schematic of a detonation spray torch [13].

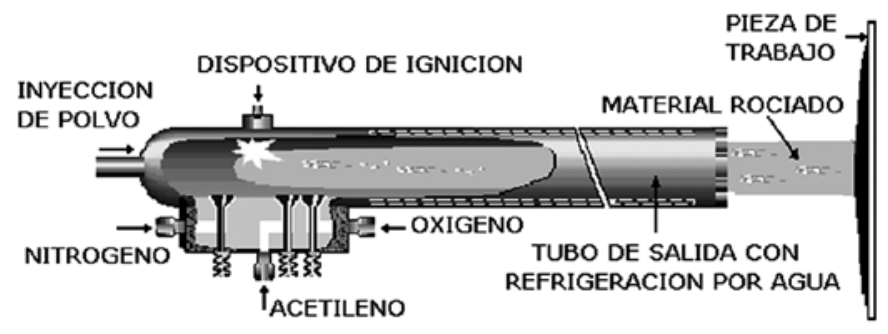

Rociado térmico por arco eléctrico: El proceso de rociado térmico por arco eléctrico, utiliza un arco voltaico para el calentamiento y la fusión de dos electrodos consumibles de alambre, cargados eléctricamente (un alambre es positivo "ánodo", el otro negativo "cátodo") que se encuentran inicialmente aislados uno del otro, siendo alimentados conjuntamente, y avanzan automáticamente para encontrarse en un punto, en una niebla de gas atomizante, donde una diferencia de potencial entre 18 y 40 $\mathrm{V}$ es aplicada a través de los alambres, iniciando un arco eléctrico controlado en su intersección, que funde y pulveriza las puntas de los alambres que funcionan como electrodos. Un gas, usualmente aire comprimido, es dirigido a través de la zona del arco, atomizando el metal fundido y proyectando las partículas sobre el substrato previamente preparado. Las partículas fundidas que impactan en el substrato solidifican rápidamente para formar una capa $[29,30]$. Un esquema del proceso de rociado térmico por arco eléctrico es presentado en la figura 4.

Figura 4. Esquema del proceso de rociado térmico por arco eléctrico [13].

Figure 4. Schematic of arc thermal spray process [13].

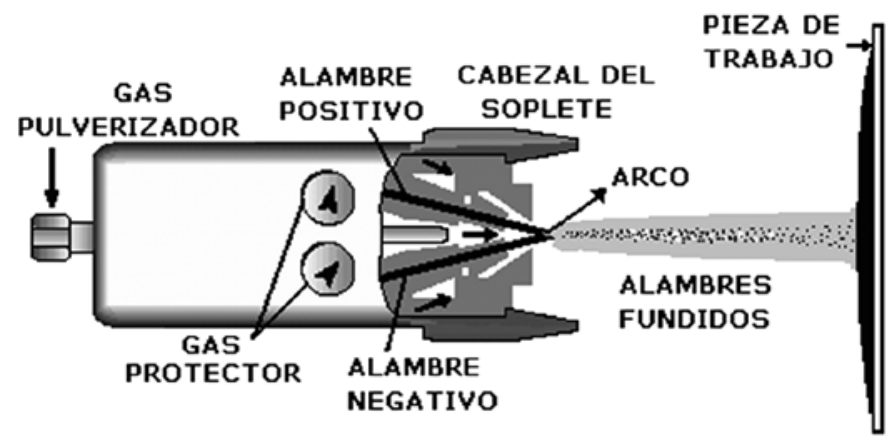

El equipo de arco pulsado se desarrolló con el fin de mejorar la calidad de las capas depositadas. En este equipo, el material de aporte en alambre es atomizado en el arco y propulsado hacia un substrato por un chorro de plasma pulsado. Este desarrollo es incitado por la observación en 
las velocidades de la partícula alcanzadas en el rociado térmico por arco tradicional, ya que son bajas para permitir la deposición de materiales con capas densas, de alta calidad, como también de la expectativa que velocidades más altas dan lugar a capas de mejor calidad. Es deseable aumentar la velocidad del material durante el rociado térmico para reducir al mínimo el enfriamiento del material en la corriente de gas, para eliminar la oxidación de las gotas fundidas y producir capas con baja porosidad [31].

Rociado térmico por plasma con arco no transferido: El proceso de rociado térmico por plasma (APS- Air Plasma Spraying) utiliza el calor de un arco que se forma dentro de la boquilla, entre un ánodo de cobre refrigerado por agua $\mathrm{y}$ un cátodo de tungsteno. El gas o la mezcla de ellos atraviesan el arco y se calienta a temperaturas elevadas (8.700 ${ }^{\circ} \mathrm{C}$ a $12.000{ }^{\circ} \mathrm{C}$ ) que parcialmente lo ioniza produciendo el plasma [32]. A la salida de la boquilla, el gas se asocia nuevamente liberando gran cantidad de calor, para fundir los materiales del recubrimiento, en forma de polvo. Este es introducido en el plasma, siendo fundido y propulsado hacia el substrato a través de un flujo de alta velocidad. El término "arco plasma" es utilizado para describir una familia de procesos que usan un arco eléctrico constreñido para suministrar energía térmica de alta densidad [33, 34]. Una antorcha de plasma es ilustrada en la figura 5.

Figura 5. Esquema de una antorcha de rociado térmico por plasma con arco no transferido [4].

Figure 5. Schematic of a torch for thermal spraying nontransferred arc plasma [4].

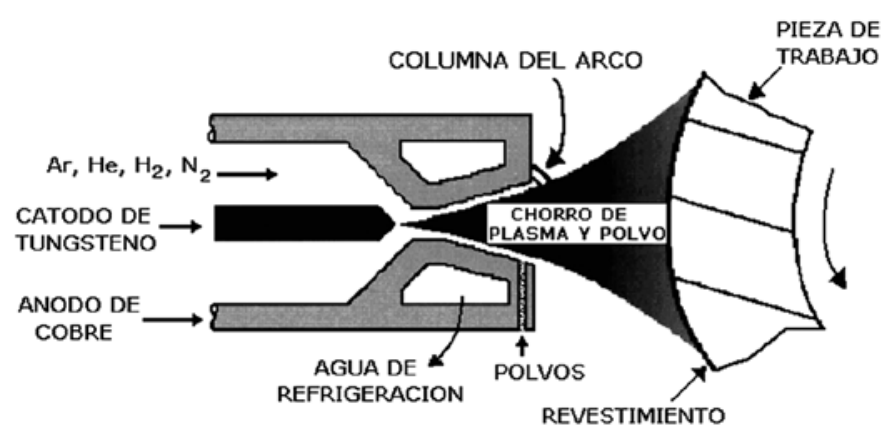

Rociado térmico por plasma con arco transferido: El proceso de rociado térmico por plasma con arco transferido, más conocido como PTA (Plasma Transferred Arc), adiciona al proceso de rociado térmico por plasma la capacidad de calentamiento y fusión superficial del substrato, siendo prácticamente una combinación de procesos de soldadura y de rociado térmico. En este proceso se genera una corriente entre el electrodo de la antorcha que actúa como cátodo y la pieza de trabajo conductora (substrato) que actúa como ánodo. Los recubrimientos obtenidos son densos, localizados y unidos metalúrgicamente al metal base. La fusión superficial y la profundidad de la penetración son controladas por la corriente del arco. El material de adición puede estar en forma de polvo o alambre [35, 36]. La figura 6 es una representación esquemática del proceso por plasma de arco transferido.

Figura 6. Esquema de rociado térmico por plasma con arco transferido $[13,35]$.

Figure 6 . Scheme thermal spraying by plasma transferred arc $[13,35]$.

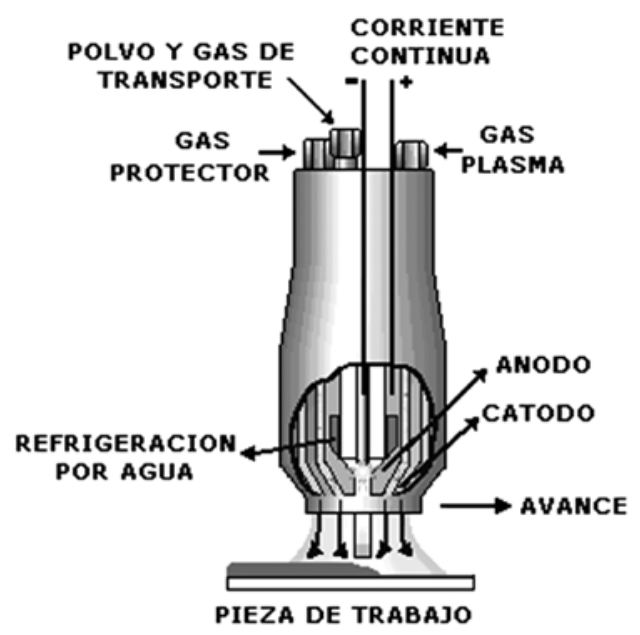

\section{VENTAJAS DEL ROCIADO TÉRMICO}

La tecnología de rociado térmico está siendo utilizado con éxito en el mundo desarrollado, aumentando la inversión en la evolución de los equipos y en el desarrollo de investigaciones para mejorar y aumentar las aplicaciones de los recubrimientos y atender los requerimientos de la ingeniería de superficies. El rociado térmico viene aumentando su reconocimiento en otros segmentos industriales, como en la industria automovilística, electrónica y petroquímica, entre otras [37]. El uso de revestimientos cerámicos duros sobre componentes, como en el caso de extrusoras de tubos plásticos, fabricados en acero inoxidable, es de gran importancia y versatilidad en la recuperación de piezas o en el aumento de la vida útil de estos componentes y equipos. En muchas industrias utilizan el rociado térmico para aumentar el funcionamiento y reducir costos de producción y de mantenimiento, en ambientes altamente erosivos o corrosivos, además de ser una técnica que puede reemplazar procesos de manufactura contaminantes como el cromo duro [38]. La figura 7, muestra la forma como llegan y como se terminan los émbolos de industria petrolera para la reconstrucción y fabricación por rociado térmico con aleaciones auto fundentes Ni-Cr-B-C.

Los recubrimientos aplicados por rociado térmico son rentables y sirven para agregar características y calidades superiores de funcionamiento a una superficie. La variedad de capas que se pueden obtener por rociado térmico 
son virtualmente ilimitadas. Los recubrimientos pueden ser metálicos, cerámicos, poliméricos o de cualquier combinación deseada para dar un amplio rango de características físicas. Si bien estos métodos de rociado térmico están siendo usados con éxito en el mundo desarrollado, a un no se tiene una completa compresión de los procesos metalúrgicos que tienen lugar durante el rociado térmico para los múltiples substratos a los que se aplican estos recubrimientos, dado básicamente a que estos se forman por unión partícula-partícula del metal aportado, resultando una capa superficial cuya estructura es altamente anisotrópica y la evaluación de sus propiedades físicas y mecánicas resulta compleja $[40,41]$.

Figura 7. Reconstrucción de émbolos de bomba hidráulica de la industria petrolera por rociado térmico con aleaciones auto fundentes Ni-Cr-B-C [39].

Figure 7. Reconstruction of hydraulic piston pump the oil industry by thermal spray using self fluxing alloys Ni-CrB-C [39].
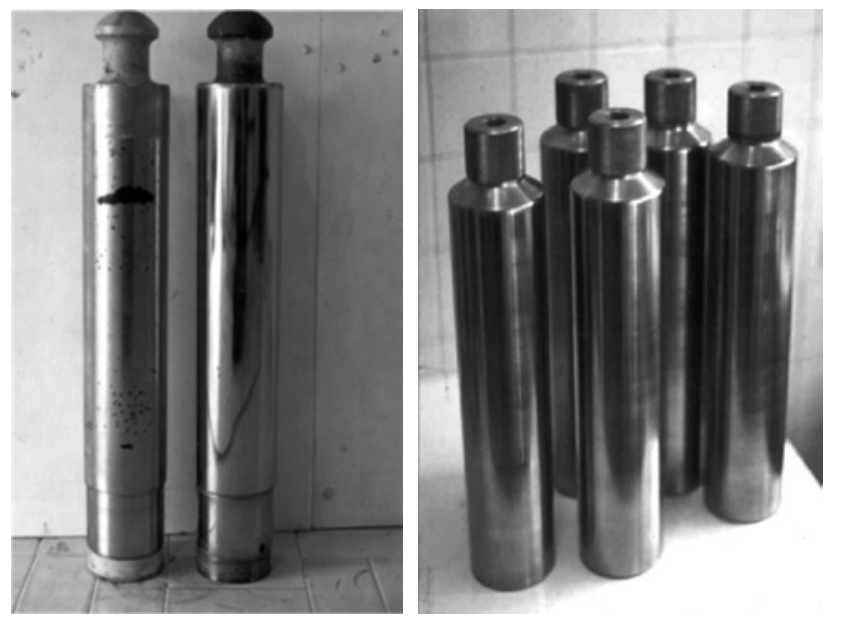

Numerosos componentes son sometidos a erosión-corrosión, en donde la velocidad de degradación de los aceros de baja aleación en estos entornos no son aceptables. El uso de paredes más gruesas y aceros de alta aleación para prolonga la vida útil de los componentes, es una alternativa muy costosa. Una solución es cubrir por rociado térmico los aceros de baja aleación con un material resistente a la erosión-corrosión como las aleaciones de tipo WC-Co, WC-Co-Ni, WC-Co-Cr, Ni-Cr-B-Si, entre otras [42, 43]. En la industria siderúrgica hay fuertes desgastes por fricción del tipo metal-metal, en donde las piezas como las bobinadoras de trefilado se desgastan rápidamente. La recuperación por medio del procedimiento de rociado térmico por arco eléctrico casi le duplica la vida útil de bobinado pasando de 130 días a 230 días de operación, además de reducir el tiempo empleado en la reparación de la pieza. En la figura 8 se observa cómo se recupera una bobina de trefilado de hierro colado gris por este método.
Las ventajas de la aplicación de revestimientos de zinc, por rociado térmico, en puentes, para la protección contra la corrosión atmosférica y/o marítima. A pesar de que sean altos los costos de la aplicación de estos revestimientos, aproximadamente 1,5 veces mayor que los de sistemas de pintura de alto desempeño, los respectivos costos del ciclo de vida son menos de tres cuartos de los costos de sistemas de pintura, justificando su uso. La ventaja de la aplicación de materiales por rociado térmico, con alambres tubulares es la posibilidad de formular las propiedades físicas y metalúrgicas requeridas en la composición de los polvos dentro del alambre $[44,45]$.

Figura 8. Recuperación de una bobina de trefilado de hierro colado gris, por medio de rociado térmico por arco [39].

Figure 8. A coil recovery of gray cast iron drawn through arc thermal spray [39].

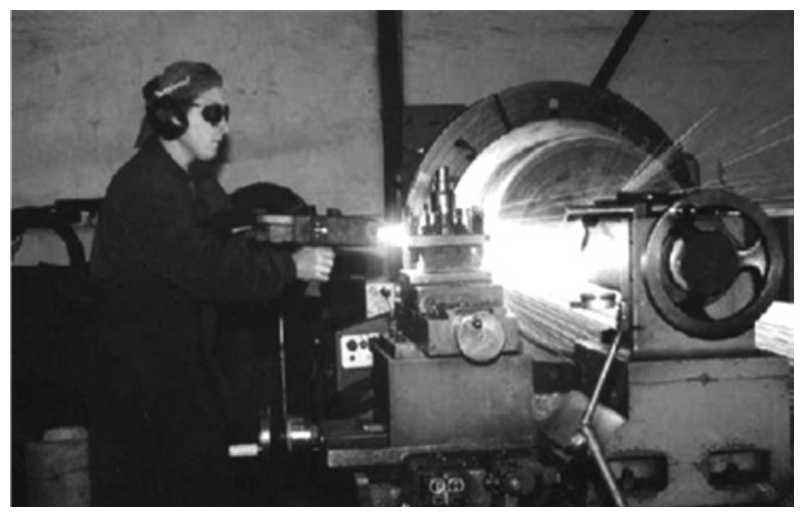

El crecimiento de la industria del rociado térmico, en los parámetros de la industria de soldadura, irá a demandar mayor inversión en entrenamiento operacional, educación en cuanto al patronado y certificación, a fin de que los actuales y futuros usuarios puedan tener asegurados los productos de alta calidad y confianza, ampliamente aceptados, especialmente cuando nuevos procesos, aplicaciones, automatización y materiales están siendo introducidos. Por lo tanto, las aplicaciones para la tecnología del rociado térmico, dentro de patrones debidamente establecidos, es limitado, sólo, "por la imaginación" [4, 46].

\section{REVESTIMIENTOS PARA BARRERA TÉRMICA (TBC)}

Una de las grandes aplicaciones de los revestimientos cerámicos por rociado térmico por plasma es el revestimiento para barrera térmica, conocido como TBC (Thermal Barrier Coating). Esos revestimientos son aplicados, para la protección de componentes metálicos que sufren degradación debido a corrosión, oxidación o sobrecarga de calor excesivo durante el servicio en ambientes térmicamente drásticos [47, 48, 49]. Su principal aplicación es en motores diesel, turbinas a gas y en las industrias automovilística y 
aeronáutica, otra aplicación es en el sector de generación de energía. Los TBCs se usan en los componentes en la sección caliente de la turbina, bajando conductividad térmica de estos materiales y facilitando el funcionamiento, disminuyendo la temperatura de los componentes en presencia de combustión a alta temperatura. En las turbinas a gas, de la industria aeronáutica, los TBC pueden ser más eficientes debido a la reducción de la temperatura de operación del sustrato o aumento de la temperatura del gas en operación. Así, una capa de 0,30 mm de espesor de aislante por TBC puede reducir la temperatura de componentes de la turbina en hasta $300^{\circ} \mathrm{C}$. Esta gran reducción de temperatura puede aumentar las capacidades de trabajar a más altas temperaturas de los componentes, aumentando su eficiencia, así como su durabilidad. Los revestimientos de barrera térmica en motores diesel, protegen los substratos metálicos contra la corrosión y la oxidación a alta temperatura. Reducen las temperaturas de los substratos y los efectos de la fatiga térmica y la radiación ayuda a convertir más calor en energía útil [50, 51, 52].

\section{CONCLUSIONES}

- Los recubrimientos por rociado térmico aumentan la vida útil de equipos y maquinaria, al obtener altos rendimientos durante más tiempo y reducir el número de fallas. La tecnología del rociado térmico es uno de los medios más eficaces que se hayan desarrollado para combatir el desgaste prematuro, al adelantarse a la falla o al momento en que el equipo o elemento deja de trabajar en sus condiciones óptimas. De esta forma se optimiza la disponibilidad del equipo, se disminuye costos de mantenimiento y se maximiza la vida útil.

- El rociado térmico es una técnica que ha mostrado buenos resultados en la recuperación de piezas, con lo cual se puede mejorar la forma de proteger las estructuras y de reducir costos de mantenimiento, aumentando la productividad de los equipos.

- Los costos debido a la corrosión y el desgaste son muy altos en el ámbito mundial, por lo que se necesitan alternativas para mitigar este problema y el proceso de rociado térmico ha demostrado ser muy útil para la protección contra la corrosión y el desgaste.

- Los progresos la tecnología del rociado térmico tienen gran aceptación en la industria, tanto en la fabricación de piezas como en el mantenimiento, en donde el campo de aplicación se amplía cada vez más debido al desarrollo de nuevas aleaciones y procesos.

\section{REFERENCIAS}

[1] Zhenyu Z. b., Zhiping W. \& Bunv L. (2009). Wear characterization of thermal spray welded $\mathrm{Ni}-\mathrm{Cr}-\mathrm{B}-\mathrm{Si}-\mathrm{RE}$ alloy coatings. Journal of materials processing technology 209 (31), 1368-1374.
[2] Shenglin L., Xueping Z. \& Gangqiang G. (2010). Dry sliding wear behavior and corrosion resistance of NiCrBSi coating deposited by activated combustion-high velocity air fuel spray process. Materials and Design 31: 913-917.

[3] Vázquez, A. \& Damborenza, J. (2001). Ciencia e ingeniería de la superficie de los materiales metálicos. Madrid. Textos Universitarios. CENIM.

[4] Trevisan, R. \& Lima, C. (2002). Aspersao Termica Fundamentos E Aplicacoes. Sao Paulo: Editorial Artliber.

[5] Marulanda, J., Zapata, A. \& Isaza, E. (2007). Protección contra la corrosión por medio del rociado térmico. Scientia Et Technica. No 34: 237 - 243.

[6] Zhang, H., Wang, X., Zheng, Z. \& Jiang, X. (2001). Studies of splat morphology and rapid solidification during thermal spraying. International Journal of heat and mass transfer 44 (24), 4579-4592.

[7] Gonzales, S. (2006). Caracterización microestructural y mecánica de barreras térmicas por APS y EB-PVD degradadas por fatiga térmica y por contacto. Barcelona. Universidad Politécnica de Cataluña.

[8] Yang, K., Fukumoto, M., Yasui, T. \& Yamada, M. (2013). Role of substrate temperature on microstructure formation in plasma-sprayed splats. Surface $\mathcal{E}$ Coatings Technology 214: 138-143.

[9] Zirari, M., Abdellah El-Hadj, A. \& Bacha, N. (2010). Numerical analysis of partially molten splat during thermal spray process using the finite element method. Applied Surface Science 256 (11), 3581-3585.

[10] Tariq S., Chunmei X. \& Pravansu M. (2007). Modeling and analysis of combustion assisted thermal spray processes. International Journal of Thermal Sciences 46 (8): 755-767.

[11] Cinca, N., Camello C. \& Guilemany, J. (2013). An overview of intermetallics research and application: Status of thermal spray coatings. Journal of Materials Research and Technology 2 (1), 75-86.

[12] Davis. J. (2004). Handbook of Thermal Spray Technology. Ohio: Thermal Spray Society and ASM. International.

[13] Linspray. (2009). Proyección térmica de superficies. Abello Linde. Catalogo consultado en Diciembre del 2012. Disponible en: http://www.abellolinde.es/internet.lg.lg. esp/es/images/LINSPRAY\%20proyecci\%C3\%B3n\%20 t \% C3\% A 9 rmica $\% 20$ de $\% 20$ superficies $\% 2013049$ 0207316_60056.PDF. 
[14] Fincke, J.R., Swank, W.D., Bewley, R.L., Haggard, D.C., Gevelber, M. \& Wroblewski, D. (2001). Diagnostics and control in the thermal spray process. Surface and Coatings Technology 146-147: 537-543.

[15] Yandouzi, M., Sansoucy, E., Ajdelsztajn, L. \& Joduin, B. (2007). WC- Based cermet coating produce by cold gas dynamic and pulse gas dynamic spraying processes. Surface \& Coatings Technology 202 (2), 382-390.

[16] Santa, J.F., Espitia, L.A., Blanco, J.A., Romo, S.A. \& Toro, A. (2009). Slurry and cavitation erosion resistance of thermal spray coatings. Wear 267 (1-4), 160-167.

[17] Mingheng L., Dan S. \& Panagiotis D. C. (2005). Modeling and control of HVOF thermal spray processing of WC-Co coatings. Powder Technology 156: 177-194.

[18] Pombo M. H., Paredes, S. C., Schereiner H. W. \& Calixto A. (2007). Comparison of aluminum coatings deposited by flame spray and by electric arc spray. Surface $\mathcal{E}$ Coatings Technology 202 (1), 172-179.

[19] García, F. \& Cuetos, J. (2001). Recubrimientos de proyección por plasma. Universidad de Oviedo. Encuentro de tribología. Septiembre 20 y 21.

[20] Gonzalez, R., Cadenas, M., Fernández, R., Cortizo, J.L. \& Rodríguez E. (2007). Wear behavior of flame sprayed $\mathrm{NiCrBSi}$ coating remelted by flame or by laser. Wear 262 (3-4), 301-307.

[21] Singrathaia S., Rachpecha, V. \& Niyomwas, S. (2011). A Thermal Coating Process Using Self-Propagating HighTemperature Synthesis Assisted Flame Spray Coating Process. Energy Procedia 9: 398-403.

[22] Redjdala, O., Zaidb, B., Tabtic, M., Hendad, K. \& Lacaze, P. (2013). Characterization of thermal flame sprayed coatings prepared from $\mathrm{FeCr}$ mechanically milled powder. Journal of Materials Processing Technology 213: 779-790.

[23] Jalali, A. M., Norouzi, H. S., majd, M., Talebi H \& Ghamari A. (2010). Application of HVOF Thermal Spraying in High Speed Gas Compressor Shafts. World Academy of Science, Engineering and Technology 45 (45), 360-365.

[24] Bolellia,G., Bonferronia, B., Laurilab, J., Lusvarghia, L., Milantia, A., Niemib, K. \& Vuoristob, P. (2012). Micromechanical properties and sliding wear behaviour of HVOF-sprayed Fe-based alloy coatings. Wear 276-277: 29-47.

[25] Mostaghimi, J., Chandra, S., Ghafouri-Azar, R. \& Dolatabadi, A. (2003). Modeling thermal spray coating pro- cesses: a powerful tool in design and optimization. Surface and Coatings Technology 163-164: 1-11.

[26] Dongmo E., Wenzelburger, M. \& Gadow R. (2008). Analysis and optimization of the HVOF process by combined experimental and numerical approaches. Surface $\mathcal{E}$ Coatings Technology 202 (18), 4470-4478.

[27] Maranho, O., Rodríguez, D. Boccalini M. \& Sinatora A. (2008). Influence of parameters of the HVOF thermal spray process on the properties of multicomponent white cast iron coatings. Surface \& Coatings Technology 202 (15), 3494-3500.

[28] Saravanan, P., Selvarajan, V., Rao D.S., Joshib, S.V. \& Sundararajan, G. (2000). Influence of process variables on the quality of detonation gun sprayed alumina coatings. Surface and Coatings Technology 123 (1), 44-54.

[29] Planche, M.P., Liao, H. \& Coddet, C. (2004). Relationships between in-flight particle characteristics and coating microstructure with a twin wire arc spray process and different working conditions. Surface and Coatings Technology $182(2-3), 215-226$.

[30] Gedzevicius, I. \& Valiulis, A.V. (2006). Analysis of wire arc spraying process variables on coatings properties. Journal of Materials Processing Technology 175 (1-3), 206-211.

[31] Fanga, J.C., Xub, W.J. \& Zhao, Z.Y. (2005). Arc spray forming. Journal of Materials Processing Technology 164-165: 1032-1037.

[32] Brossard, S., Munroe, P., Tran, A. \& Hyland, M. (2010). Study of the effects of surface chemistry on splat formation for plasma sprayed $\mathrm{NiCr}$ onto stainless steel substrates. Surface \& Coatings Technology 204:1599-1607.

[33] Nusair, K. \& Lu, J. (2009). Manipulation of air plasma spraying parameters for the production of ceramic coatings. Journal of materials processing technology 209 (5), 2508-2514.

[34] Karger, M., Vaßen, R. \& Stöver, D. (2011). Atmospheric plasma sprayed thermal barrier coatings with high segmentation crack densities: Spraying process, microstructure and thermal cycling behavior. Surface $\mathcal{E}$ Coatings Technology 206 (1), 16-23.

[35] Gatto, A., Bassoli, E. \& Fornari M. (2004). Plasma Transferred Arc deposition of powdered high performances alloys: process parameters optimization as a function of alloy and geometrical configuration. Surface $\mathcal{E}$ Coatings Technology 187 (2-3), 265-271. 
[36] Yugeswarana, S., Kobayashi, A., Ananthapadmanabhanb, P.V. \& Lusvarghi, L. (2011) Influence of processing variables on the formation of $\mathrm{La}_{2} \mathrm{Zr}_{2} \mathrm{O}_{7}$ in transferred arc plasma torch processing. Current Applied Physics 11: Issue 6. 1394-1400.

[37] Sheir, L., Jarman, R. \& Burstein, G. (2000). Corrosion. Corrosion Control. Volume 2. Oxford: Butterworth-Heinemann: 446-461.

[38] Aruna, S., Balaji, N., Shedthi, J. \& Grips W. (2012). Effect of critical plasma spray parameters on the microstructure, microhardness and wear and corrosion resistance of plasma sprayed alumina coatings. Surface $\mathcal{E}$ Coatings Technology 208: 92-100.

[39] Sager. (1998). Tecnología de Soldaduras para Mantenimiento y Reparación. Bogotá. Eutectic-Castolin.

[40] Laribi, M., Mesrati, N. Vannes, A.B. \& Treheux, D. (2003). Adhesion and residual stresses determination of thermal sprayed molybdenum on steel. Surface and coating Technology 166 (2-3), 206-212.

[41] Toma, D., Brandl, W. \& Marginean, G. (2001). Wear and corrosion behavior of thermally sprayed cermet coatings. Surface and Coatings Technology 138 (2-3), 149-158.

[42] Hjornhede, A. \& Nylund, A. (2004). Adhesion testing of thermally sprayed and laser deposited coating. Surface and coating technology, 184 (2-3), 208-218.

[43] García, A., Cadenas, M., Fernández, M. \& Noriega, A. (2013). Tribological effects of the geometrical properties of plasma spray coatings partially melted by laser. Wear 305 (1-2), 1-7.

[44] Chaliampalias, D., Vourlias, G., Pavlidou, E., Stergioudis, G., Skolianos, S. \& Chrissafis, K. (2008). High temperature oxidation and corrosion in marine environments of thermal spray deposited coatings. Applied Surface Science 255 (5), 3104-3111.

[45] Niranatlumpon, P. \& Koiprasert, H. (2006). Improved corrosion resistance of thermally sprayed coating via surface grinding and electroplating techniques. Surface $\mathcal{E}$ Coatings Technology 201 (3-4), 737-743.

[46] Echavarria, A. (2003). Una perspectiva de los biomateriales vista desde un diseño específico: La prótesis total de cadera. DYNA 30: 95-108.

[47] Basu, S., Ye, G., Gevelber, M. \& Wroblewski, D. (2005). Micro crack formation in plasma sprayed thermal barrier coatings. International journal of refractory metals and hard materials 23 (4-6), 335-343.

[48] Boulos, M., Jiang X. \& Gitzhofer, F (2006). Thermal Spray Coating of Tungsten for Tokomak Device. Plasma Science and Technology 8 (2), 164-167.

[49] Haynes, J.A., Lance, M.J., Pint, B.A. \& Wright, I.G. (2001). Characterization of commercial EB-PVD TBC systems with CVD $(\mathrm{Ni}, \mathrm{Pt}) \mathrm{Al}$ bond coatings. Surface and Coatings Technology 146-147: 140-146.

[50] Liangde X., Xinqing M., Jordan, E.H., Padture,N.P., Xiao, D.T. \& Gell, M. (2004). Deposition mechanisms of thermal barrier coatings in the solution precursor plasma spray process. Surface and Coatings Technology 177 (5), 103-107.

[51] Rico, A., Gómez, J., Múnez, C.J., Poza, P. \& Utrilla, V. (2009). Mechanical properties of thermal barrier coatings after isothermal oxidation. Depth sensing indentation analysis. Surface \& Coatings Technology 203 (16), 2307-2314.

[52] Curry, N. \& Donoghue, J. (2012). Evolution of thermal conductivity of dysprosia stabilised thermal barrier coating systems during heat treatment. Surface $\mathcal{E}$ Coatings Technology 209: 38-43. 